\title{
Match Field based Algorithm Selection Approach in Hybrid SDN and PCE Based Optical Networks
}

\author{
${ }^{1}$ P.Selvaraj, ${ }^{2}$ V.Nagarajan \\ ${ }^{1}$ Research Scholar, SRM University, School of Computing, \\ Kattankulathur, India \\ [e-mail: selvaraj_mail@yahoo.com] \\ ${ }^{2}$ Consultant, SRM University, School of Computing, \\ Kattankulathur, India \\ [e-mail: nagge2000@yahoo.co.in] \\ *Corresponding author: P.Selvaraj
}

Received May 18, 2017; revised November 20, 2017; revised February 19, 2018; revised April 17, 2018; revised June 10, 2018; accepted August 6, 2018; published December 31, 2018

\begin{abstract}
The evolving internet-based services demand high-speed data transmission in conjunction with scalability. The next generation optical network has to exploit artificial intelligence and cognitive techniques to cope with the emerging requirements. This work proposes a novel way to solve the dynamic provisioning problem in optical network. The provisioning in optical network involves the computation of routes and the reservation of wavelenghs (Routing and Wavelength assignment -RWA). This is an extensively studied multi-objective optimization problem and its complexity is known to be NP-Complete. As the exact algorithms incurs more running time, the heuristic based approaches have been widely preferred to solve this problem. Recently the software-defined networking has impacted the way the optical pipes are configured and monitored. This work proposes the dynamic selection of path computation algorithms in response to the changing service requirements and network scenarios. A software-defined controller mechanism with a novel packet matching feature was proposed to dynamically match the traffic demands with the appropriate algorithm. A software-defined controller with Path Computation Element-PCE was created in the ONOS tool. A simulation study was performed with the case study of dynamic path establishment in ONOS-Open Network Operating System based software defined controller environment. A java based NOX controller was configured with a parent path computation element. The child path computation elements were configured with different path computation algorithms under the control of the parent path computation element. The use case of dynamic bulk path creation was considered. The algorithm selection method is compared with the existing single algorithm based method and the results are analyzed.
\end{abstract}

Keywords: Software Defined Networking-SDN, Algorithm Selection, Path Computation Element-PCE. 


\section{Introduction}

The network operators are striving to maintain reliable network work connectivity between the applications, cloud servers and data centers. The performance of the network greatly depends on the type and capacity of the network which connects users with the application. To maintain the required QoS/user satisfaction, the end-to-end links must be maintained in a more reliable manner. To achieve such reliability the network must be provisioned, monitored and maintained with high-capacity, low-latency, jitter-free connections. All parts of the end to end information transport are likely to exploit optical fibers and lasers; including $100 \mathrm{Gbps}$ access networks, 10 Gbps local area networks, and even 1 Gbps to the desktop. The next generation optical technologies must cope with the bandwidth needs of the future services that do not even exist yet but recent history indicates are sure to emerge. To meet the evolving computing and communications needs, a 'tera-era' vision is proposed. The fundamental advances towards the 'tera-era' has started exploiting the optical technologies and its associated control plane algorithms. To manage the dynamic high data rate and high bandwidth data transmission, the optical backbone network has to be provisioned and managed effectively. At present, the IP and optical layers have been managed independently to deal with any dynamic network scenarios. With the emergence of software-defined networking (SDN), the control plane can be isolated and managed from a central location. This consistent partitioning of layered functionalities with fine-grained control and management plane abstraction has empowered the network operators to offer a new range of administration in an on-demand and agile manner. The SDN enables the programmability of data and control plane through the well defined API's [1]. With SDN, the exact provisioning and optimal performance can be realized in optical transmission.

The objective of this paper is to exploit SDN-based controlling ability to realize the dynamic path provisioning ability in optical communication. This proposed work intends to offer high throughput with decreased latency. The SDN is used in controlling frameworks that requires global end-to-end network visibility, and flexibility in terms of fine-grained resource dissemination. It is broadly acknowledged that the SDN-based optical networking can improve the QoS needs of the paths in a more successful manner. In SDN-based optical networking, the controller will take the critical decisions on how to configure and when to reconfigure the set of optical connections with the intention to preserve the various QoS necessities like bandwidth, BER, congestion, delay of the dynamic traffic [2]. Hence the SDN controller can dynamically decide the routing, and wavelength allocation approach/algorithm to optimally take advantage of the fiber capacity and the network status.

This work proposes a unique and yet effective SDN-based mechanism for the appropriate selection of optical path computation approach. An SDN inspired, match-fields based mechanism that maps the features of network traffic with a particular algorithm, was proposed. This approach can substantially minimize the operational cost involved with the setting up of optical paths. This approach exploits the capabilities of various path computation approaches/algorithms/heuristics. As every path computation algorithm is capable of exploring the various solution space, the traffic will be distributed in a more optimal manner. In this work a software-defined controller with multiple child PCE was created in the ONOS tool. The PCE implementation has been evolved with sophisticated interfaces to manage the 
optical domains. The PCE underneath the control of the controller was made to act as the parent PCE or orchestrator. The child PCEs with handpicked optical path computation algorithms were configured and made to function beneath the parent PCE. The traffic needs with different QoS requirements were dynamically created with IPERF tool. And the different types of demands were associated with unique child PCEs in real time. The traffic requirements with specific constraints were matched with an appropriate optical path computation algorithm. And the different child PCEs were made to control the path computation process beneath the control of the parent PCE. Both the child PCEs and parent PCE were made to operate in the same domain. Hence a novel packet matching feature was proposed as an amicable method for the SDN based optical networking. The performance the established optical pipes has been observed in the ONOS tool by analyzing the traffic monitoring logs.

This paper is organized as follows: The Section.2 provides the benefits of the hybrid PCE and SDN based control mechanism along with its architectural details. The Section.3 briefly explains about the routing and wavelength assignment problem. The Section. 4 reviews about the flow table driven approach of SDN. The Section.5 explains a novel match fields based algorithm selection method. The Section. 6 and 7 discusses the simulation study and its results and finally Section. 8 concludes the paper.

\section{Hybrid SDN and PCE Based Networking}

\subsection{Software Defined Networking-SDN}

The conventional networking protocols that were developed by using data networking technologies may not be suitable for optimal inter-networking. The internet backbone has to transport multiple type and sizes of packets on top of the IP protocol. The coverage of compatibility and the management schemes are different in fundamental routing protocols, algorithms and technologies. The information transport, routing, and switching functions of software enabled protocols are considered as the core module in the evolving next generation intelligent optical networks. In such intelligent optical network, the paths must be automatically activated from the end user's end point triggered with the point and click interface. The SDN controller focuses on the lifting the essential control functions from the corresponding network elements to the centralized control plane.

The SDN was mainly evolved to overcome the issues with traditional traffic management. With the central control and management the SDN based approach, can considerably reduce the quantity of adaptive exchange of TE-Traffic Engineering messages among the peer networking devices. With SDN paradigm, the network control plane can be decoupled from the network data plane. This logical separation promotes innovative traffic monitoring and management capabilities and enables the network operators to manage and operate their networks in a more effective manner. The concept of SDN involves the physical separation of the network control and forwarding planes. The OpenFlow as a South-Bound Interface-SBI abstracts the physical and virtual data forwarding planes. The OpenFlow will lift the control plane protocol that resides inside the switching devices and keeps it in the controller's end through the well defined API's. Fig. 1. 


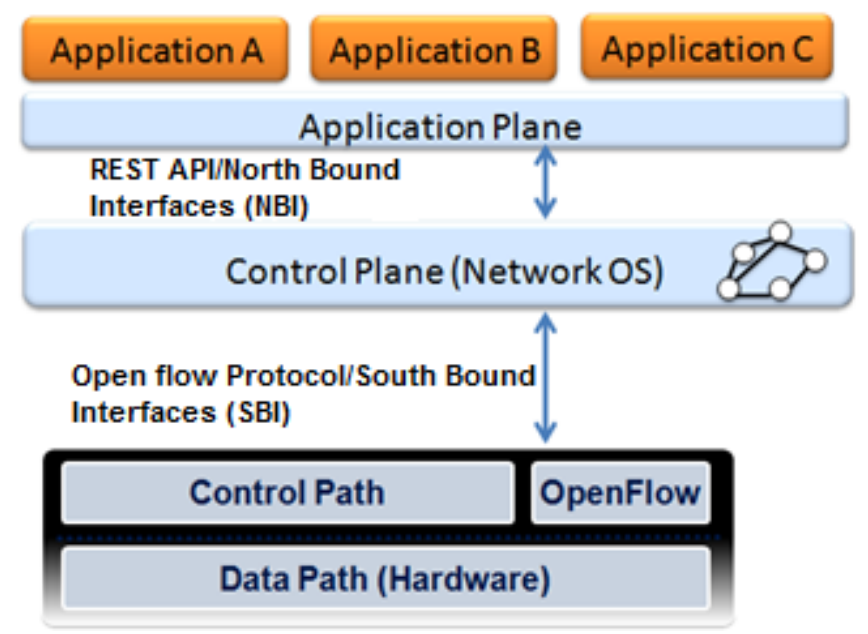

Fig. 1. SDN architecture

The SDN has been evolved from the inspiration of datacenter networking. The effective transmission capacity reservation was the promising methodology to accomplish substantial QoS in datacenters. The datacenter management normally involves multipath approach to keep up throughput efficiency. The QoS necessities of the datacenters facilitating various applications could be effectively accomplished through SDN engineering. The ability of Openflow empowered switches in handling the powerful QoS mandates had been proven with many test bed experiments. The OpenFlow gives a standard interface to control the low level switch activities. At present there are noticeable varieties of OpenFlow switches available in the market. These switch varieties gives a comprehensive knowledge to the SDN application designers to design innovative QoS based SDN systems.

With SDN a controllable framework can be realized to meet the framework level quality prerequisites in less OPEX. The SDN will enhance the flexibility and dexterity of the optical data plane by lifting the control plane from all the optical interconnection gadgets and oversee from a central server. The centralization and virtualization are the key ideas in SDN. The SDN design has the capacity to control edge and core devices from a centralized location in a programmable manner. The SDN design itself will thus give a global network wide view and the capacity to deal with the network wide actions. The cost effectiveness of SDN in optical path provisioning system was demonstrated in [3].

The features of SDN involves the following:

- Directly programmable with logical abstraction of planes

- Promotes agile control and forwarding methods

- Centrally managed with sophisticated capabilities

- Programmatically configured and controlled with automation

- Open standards-based and vendor neutral

Thus the information of traffic plane conveying about payload can be monitored by an overlaying control or management or signaling plane. From the remote end, the application plane will issue the necessary support for the management of network events. The dynamic re-configurability and global network prominence were derived from SDN with 
its optimal level of convergence. The dynamic reconfiguration,flexibility and adaptability are the essential ingredients for the evolving next generation optical networks.

\subsection{Path Computation Element-PCE under SDN Orchestration}

The SDN controller consists of a functional block known as PCE as one of its application. The PCE is an entity which computes the paths primarily based on the network graph and the required constraints [4]. The PCE can compute the paths with the updated real-time topology maintained with the TED. The PCE can be either embedded inside a routing element or it could function as a separate principal entity. In optical networks, all the networking elements may not have the control /management and routing capabilities. This has led to the development of dedicated optical PCE. With SDN, the algorithm for the PCE can be dynamically configured. The child PCE with unique path computation algorithms can be created based on the real-time requirement as the SDN controller is computationally sturdy. The PCE based SDN controller ought to provide benefits including global network monitoring, optimization and so on. The distinctive PCEs with specific algorithms can be created as software entities and made to function underneath the control of the parent PCE. The parent PCE can act as an orchestrator to coordinate between more than one child PCEs when it comes to TED update/modification and end-to-end path optimization involves multiple operator domains. The OpenFlow-OF messages could be shuttled backward and forward between the SDN controller and the OF-compatible switches. Thus, the controller can realize the preferred flow through the flow table management to accommodate the dynamic flows. Hence in our proposed approach, we have assumed that the multiple child PCEs can co-exist at the same networking domain under the parent PCE. The parent PCE will coordinate between the traffic requests and the child PCEs. And the SDN controller will provide the necessary abstractions to realize the real-time path provisioning and active monitoring of the network.

\subsection{Benefits of SDN and PCE based Networking}

One of the major benefits of hybrid SDN and PCE based networking is the possibility of "intent-based routing". The intent based routing differs from the traditional networking in the manner of handling the networking elements. The concept of intent is to inform the network elements about the particular demands of the applications. The intent can be represented in terms of low latency, excessive transmission speed, high security, ten megabits per second, maximum transport quality, required bit rate and so forth. The appropriate features of the intent are infrastructure independence, portability and composeability [5]. The SDN can provide all these required qualities in a cost-effective manner. Hence in this work, we have used SDN to propose a novel flow table match field based mechanism that is amenable for an intent based routing. The application's intents can be converted into the specified set of actions with the sophisticated North Bound API's. We have created a NOX-SDN controller configured with parent PCE element to match the intend/demand with the suitable child PCE's (approach/algorithm/heuristic) to meet the demand in a most desirable and optimal manner.

\subsection{Architecture of Hybrid SDN and PCE based Networking}

The GMPLS -Generalized Multi Protocol Label Switching is the underlying protocol which governs the control plane aspects in SDN-based PCE architectures. The sketch of the 
architecture of GMPLS based PCE architecture is given in Fig. 2.

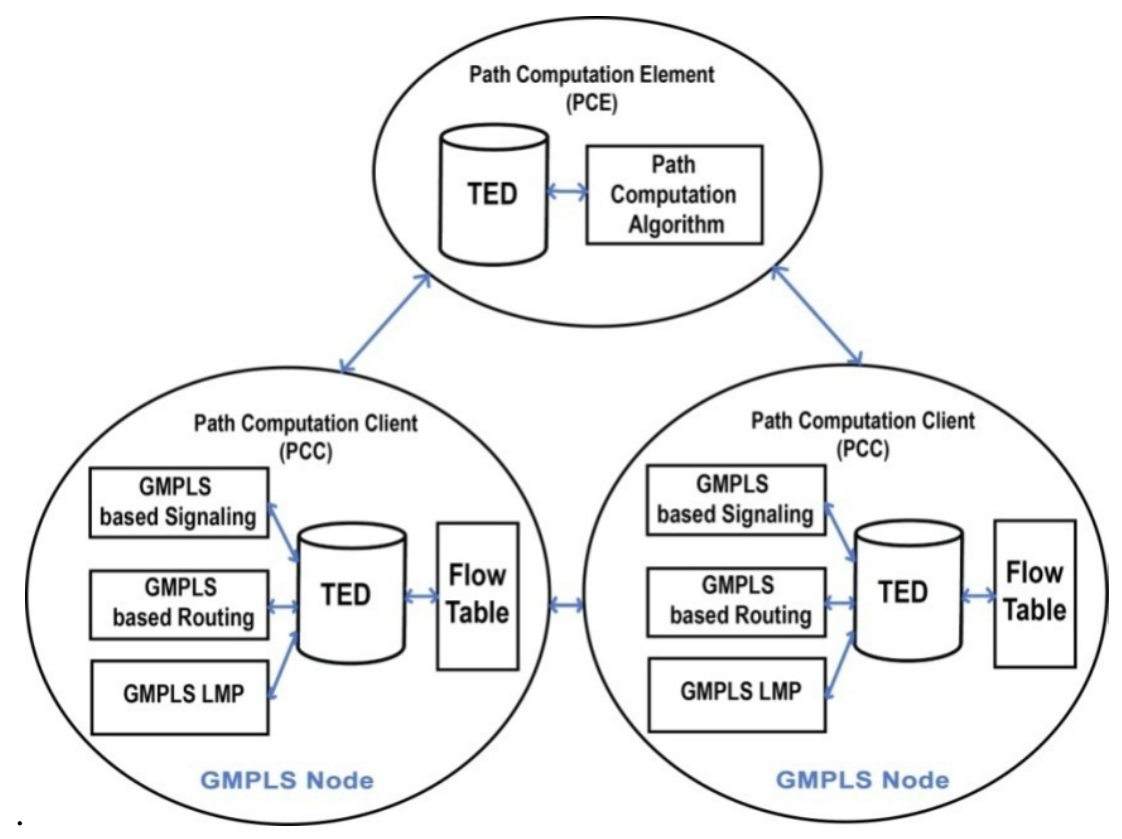

Fig. 2. PCE-based path computation architecture

With SDN controller the PCE can be programmed in real time through the interfaces [6]. The hybrid SDN based PCE can get extra benefits which include global network visibility and dynamic resource optimization with dedicated path computation abilities and so forth. The deployment architecture for the PCE-based SDN are given below Fig. 3, 4.

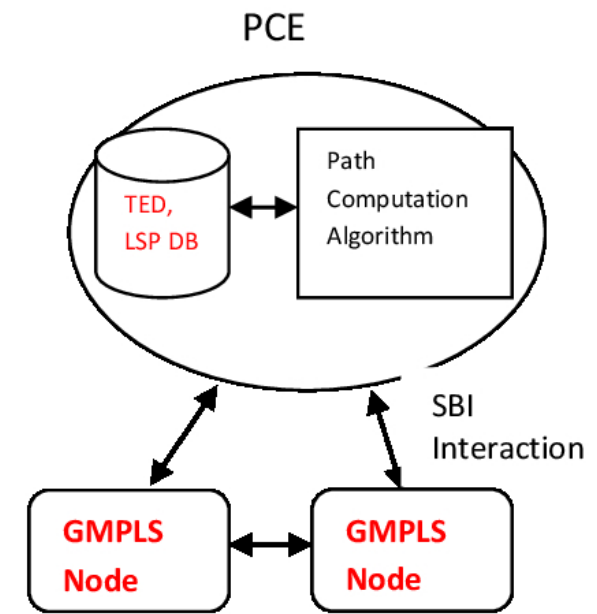

Fig. 3. Active Stateful path computation architecture

Generally, PCE is a logical functional element which is centralized or placed inside the domain or it can be distributed widely which is not an all-seeing oracle. It should coincide orderly to identify the point-to-point in a multi domain way. The protocols of the PCE path computation component is of very simple nature so as to undertake with the variety of dynamic and online information. The SDN based proposed method or scheme is constructed 
for MPLS-TE which yields an abundant of information regarding the surrounding place. Hence the SDN contoller is responsible for controlling, managing, and planning via the control plane and data plane. Therefore, it is essential to solve the problem of path computation in an optimal way.

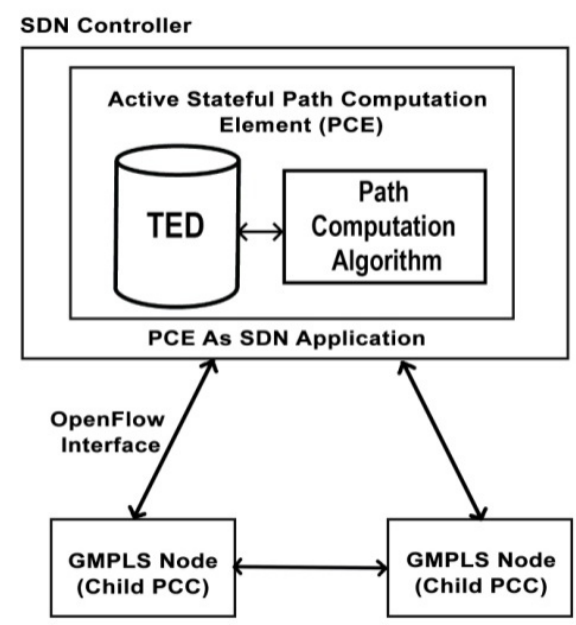

Fig. 4. Active Stateful PCE as SDN application

The path computation function may be distributed with many Path Computation Client-PCC elements or it can be centrally handled by the PCE. Such PCE can also act as an SDN application Fig. 4.

\section{Routing and Wavelength Assignment-RWA Problem in Optical Networks}

The transmission capacity of optical fiber has been gradually evolving with the advancements in DWDM technologies and the optical devices. But the number of wavelengths in a particular fiber strand is usually maintained to be limited considering the number of costlier and energy-hungry transceivers to be deployed with the optical cross-connects. The light path is the fundamental element in optical networks, which must satisfy the distinctive wavelength assignment constraint and wavelength continuity constraint during the path establishment. Apart from these basic constraints, the light path has to satisfy or optimize numerous requirements that includes delay, less congestion, and cost. The heuristics based solutions are broadly desired to resolve the RWA problem [7][8].

Some of the well known RWA algorithms for the dynamic traffic are given below: i) k-shortest path routing algorithm ii) fixed shortest path routing algorithm iii) least-congested-path routing algorithm iv) least-cost-path routing algorithm. Every RWA algorithm/heuristic has its own advantages and disadvantages. As every heuristic has its own assumptions about the operating environment. For the overall optimal functioning of the optical networks, the performance of the RWA algorithm is very crucial. The RWA algorithm applies the use of a various range of information aggregated in Traffic Engineering Database-TED through control and management protocols. Every RWA algorithm differs 
from other approaches based on its path cost function and the level of link statistics required. Because of the real-time execution requirements, the RWA algorithms have been designed with simple path cost functions that consume less TED parameters.

Most of the RWA heuristics/algorithms are having the following steps in common:

Step 1. Finding all of the potential candidate working paths

Step 2. And sorting them primarily based on the path selection policy (ex: number of Hops). For each of $k$ paths, the cost function may be calculated. (The different path cost function requires different TED parameters. The TED might be aggregated with the real-time network state (monitoring) information through the control plane protocols and NMS-Network Monitoring System. The weighted total distance might be calculated with this real-time updated information. The cost function is a function of link cost, shareability measure, and resource availability measure of the TE link).

Step 3. Finding all of the working path set \& Backup path set

Step 4. Deciding on the minimal cost path pair \{working and backup\}.

Step 5. If no route can be found, Reject the Request NO_PATH.

Step 6. Updating the path information in Label Switched Path-LSP DB and TED based on the current network status.

\section{Flow Table Matching Approach in SDN}

The OpenFlow specification resembles SDN architecture that contains an OpenFlow controller, which communicates the OpenFlow switch by using the OpenFlow protocol. One of the instantiations of SDN is OpenFlow protocol, which separates the controller and the programmable optical switch over an encrypted secure channel. The OpenFlow specification defines the components of a switch, message format, and kind of actions that need to be taken in a generalized manner. The switching functionality, ie., a packet coming from one port and switched onto some other port, was included as the 'packet-matching function' in the OpenFlow compatible switch [9][10]. Programming the packet-matching function and forwarding policies (flow table management) can be managed through the centralized OpenFlow controller. The OpenFlow uses a table-driven layout, which permits to program the behavior of OpenFlow switch using a packet-match feature.

The SDN controller can instruct the OpenFlow compatible switches about the change in flow rule by manipulating the flow tables. When the packet arrives at a switch, its header fields will be matched with the flow table entries and the appropriate action will be implemented.If any entry matches, its associated pre-defined set of actions will be performed and the counters will be updated accordingly. If the entries of the packet do not match, the switch will forward the packet to the controller. Hence whenever a packet arrives on the switch, matching against the flow table entries will be performed and the corresponding action will be taken by means of analyzing the header fields. The four general actions implemented with the aid of OpenFlow includes forward the packet to the port(s), forward the packet to the controller, drop the packet.

The OpenFlow control plane has capabilities by which the various data plane elements can be programmed using the common and standard languages with a unified instance of the 
controller [11][12]. The 'packet-matching function' and forwarding rules for the OpenFlow switch are programmed by using the OpenFlow controller. The set of standard messages that are exchanged between the OpenFlow switch and the OpenFlow controller are defined using OpenFlow protocol [13][14]. Those messages allow the controller to program the behavior in the switch Fig. 5.

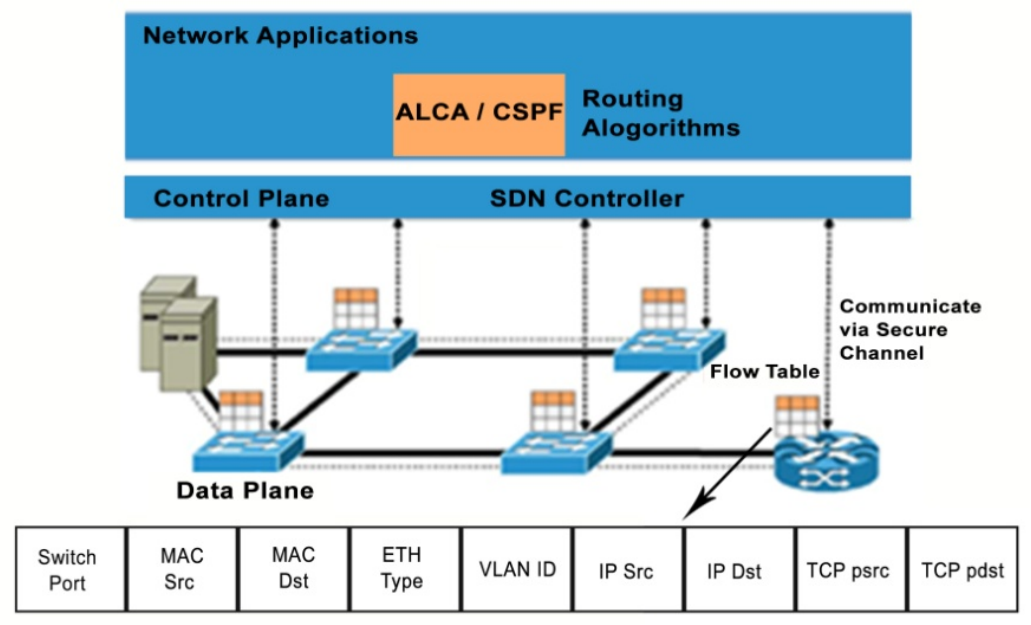

Fig. 5. SDN Controller and the Openflow compatible switch

The OpenFlow specification version1.3 defines a generic flow table that includes flow table entries like header fields, counters, and actions. Every header field corresponds to a matching field that is used to test whether or not the incoming packets belongs to that specific flow or not. The counter field provides the statistical information like the number of packets that have been forwarded or dropped and the action field permits respective action that needs to be performed for a packet matching entry. Within the packet header information, nine fields are defined on which the flow table entry might be matched as shown in the below table Table 1.

Table 1. OpenFlow protocol flow table entry fields

\begin{tabular}{|c|c|c|c|c|c|c|l|l|}
\hline $\begin{array}{c}\text { Switch } \\
\text { Port }\end{array}$ & $\begin{array}{c}\text { MAC } \\
\text { Src }\end{array}$ & $\begin{array}{c}\text { MAC } \\
\text { Dst }\end{array}$ & $\begin{array}{c}\text { ETH } \\
\text { Type }\end{array}$ & VLAN ID & IP Src & IP Dst & TCP psrc & TCP pdst \\
\hline
\end{tabular}

Generally, the OpenFlow messages fall into any one of the following three categories namely i) four symmetric messages that may be sent either by the controller or the switch, ii) fourteen controller-switch messages and iii) four async messages that may be sent from the switch to the controller. Thus the SDN has been evolved as a new network organizing technique to manage the network functions through the software-driven control plane [15][16]. Hence the SDN uses the flow table matching mechanism and it has the ability to manage the different data planes under a unified control plane algorithm. 


\section{Match Field Based RWA Algorithm Selection Approach}

This work proposes the concept of choosing between different RWA algorithm with respect to the attributes of the match fields of the traffic demand. The inspiration for this mechanism was derived from the SDN's "packet-matching feature" and its disjointness nature among the data and control plane. For every PCC request an unique algorithm will be mapped based on the signature of the traffic request, TED and the constraint(s) to be optimized. In this way, many overlay networks can be constructed to provision more than one types of services by augmenting the capabilities of many existing routing and wavelength assignment algorithms/co-operating child PCEs under the control of the parent PCE and the common TED. In this way the PCE does not have any fixed path computation algorithm. But rather many runtime PCE entities will be created based on the dynamic traffic needs. In optical networks generally, the RWA algorithm could be chosen by the technology providers based on the consideration of implementation scenarios in a fixed and static manner. Most of the implementations comply with open shortest path first approach for routing and first-fit method for wavelength assignment.

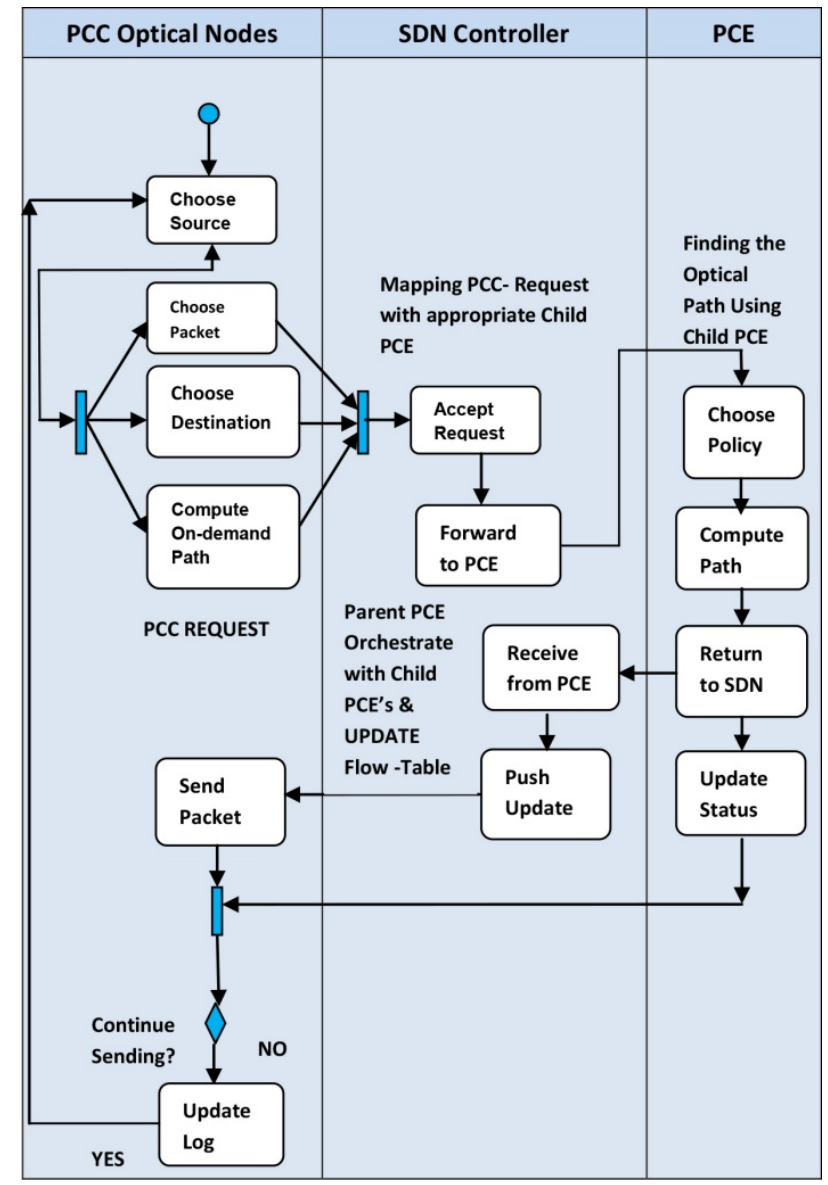

Fig. 6. The sequence of activities between the PCC, SDN Controller, Parent PCE \& Child PCE. 
The PCE can communicate with the NMS (ie., provisioning server) for the effective path computation with real-time path availability. The operator can choose the set of PCE algorithms that suits their operational requirements. Hence any time the portfolio of the path computation algorithms might also be modified according to the change in operator's policies Fig. 6, 7. In recent approaches, the constrained shortest path first (CSPF) algorithm has been widely used in the PCE based implementations. But the PCE can also associate with any of the algorithms which may also even computationally heavy. The PCE can compute many traffic engineered MPLS LSPs according to the transmission requirements.

The match-field attributes of the PCC-request and PCC-response is discussed below:

- PCC Request: Flow ID, Table ID, APP ID, Priority, Group ID, Packets, Bytes, State, Timeout, Permanent, Objective function.

- PCC Response: RP, ERO, LSPA, IRO, Signaling attributes, METRIC, BANDWIDTH.

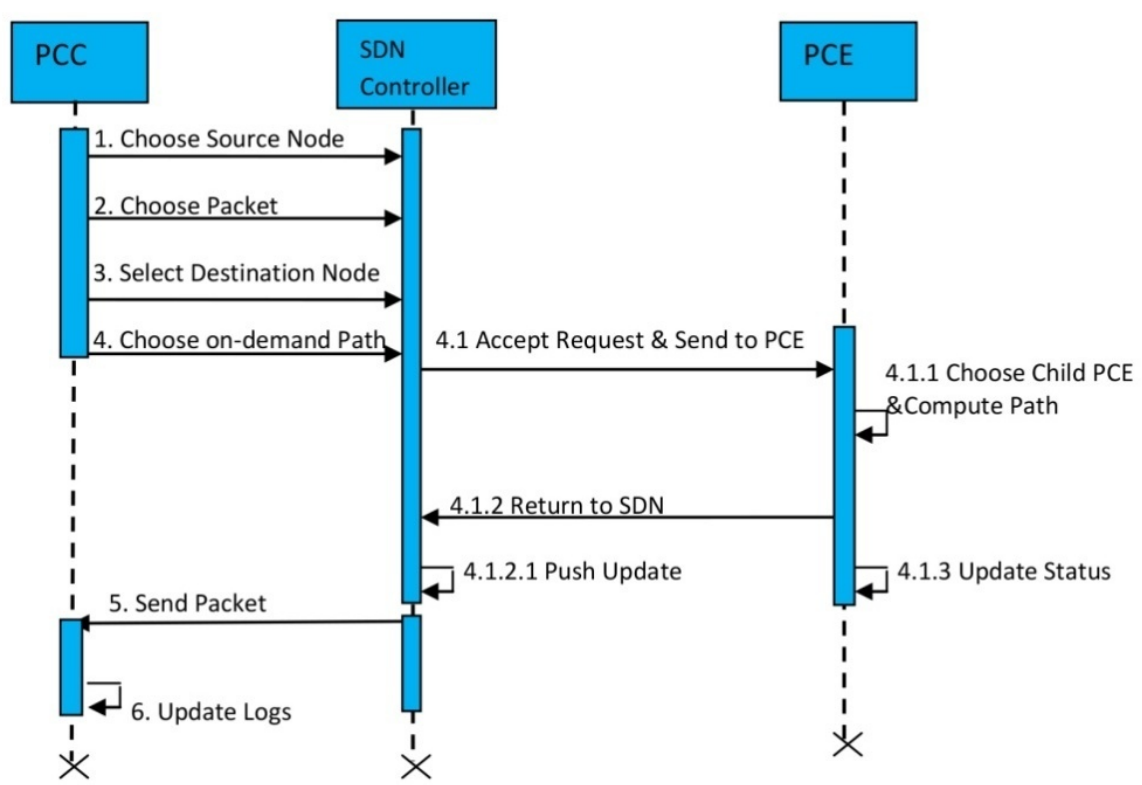

Fig. 7. The sequence of interactions between the PCC, SDN Controller, Parent PCE \& Child PCE.

The PCEP (Path Computation Element Protocol) is a session based protocol which caters the transmission of PCReq and PCRsp messages over TCP. With the updated LSP DB, the active PCE can able to advise the network with possible optimal paths for example whenever the congestion accumulates the PCE can assign flows to a newer path with different desired computed quality (jitter, delay, reliability etc.,). We have used ONOS SDN controller environment to run the desired simulation Fig. 8. The ONOS is a network operating system which manages the interfaces of SDN. The ONOS provides the API's for the control plane to manipulate the data plane in a technology and vendor independent way. Such control plane could optimize the throughout with the effective tuning of packet and optical layer attributes and promotes the abstractions like on-demand provisioning and so on [17]. All the functions of Mininet had been incorporated inside ONOS. The fundamental physical configurations of optical network can be custom designed via Mininet to emulate the physical topology. The 
ONOS-GUI panel is used to observe the network and link statistics. Through port and link monitoring the packet statistics can be observed. The PCE will acts as an application that runs on top of SDN controller through the South Bound Interface. The path provisioning can be done according to the class of service required by the flows [18]. Many flows can share a tunnel under the control of the PCEP-Tunnel provider. The tunnels can be configured according to the specified constraints.

The elements of message format, switch, and the type of actions are described by the open flow characteristics which is usually taken place in a basic type. The Open Flow protocol describes the information that are transmitted in between the open flow controller and open flow switch. The controllers are employed in programming the switch. The generic flow table comprises of a specifications like a field of header, counters, and actions that are defined by the open flow characteristics. The required steps are taken to unite the packet with that of the flow table data that are approaching the open flow switch. The flow was identified with the flow table data by the examination of incoming packets in the header fields. This uniting action of the packet data describes the action field that was performed. However, the open flow controller organizes the working table of the packet uniting functionality which is known as the packet-matching action. The modifications are made in the flow table data via the end-to-end path by the operators and providers to analyze the specific functionality.

The control plane of the open flow consists of the following cababilities:

$>$ Control plane of the open flow may appear as the separate entity from the data plane.

$>$ Different types of data plane attributes can be processed with the standard programming languages via the use of various types of interfaces.

The transmission of information within the open flow switch and the open flow controller were provided by using a protected channel of TLS-based crooked encryption. The three types of actions that are implemented in open flow switch is listed as follows:

$>$ Forward the data,

$>$ Drop, and/or

$>$ Send the messages or information.

\section{Experimental Validation}

\subsection{Optical Path Computation with Dynamic Selection of Child PCEs}

This section elaborates about the experimental steps that was followed during the simulation.

The simulation steps were broadly classified into three major divisions namely i) Conceiving the traffic demands and network status ii) deciding the action to be done iii) implementing the decision made in step ii).

\subsubsection{Conceiving the PCC request and TED status}

a) The traffic demands were generated using a poisson distribution model.

b) The real-time link and network statistics has been fed to the PCE application through the LSP-DB and TED DB. 


\subsubsection{Reasoning the Data to orient the required action}

a) Through the features of the PCC request and network the particular path computation algorithm will be pattern matched with the match fields.

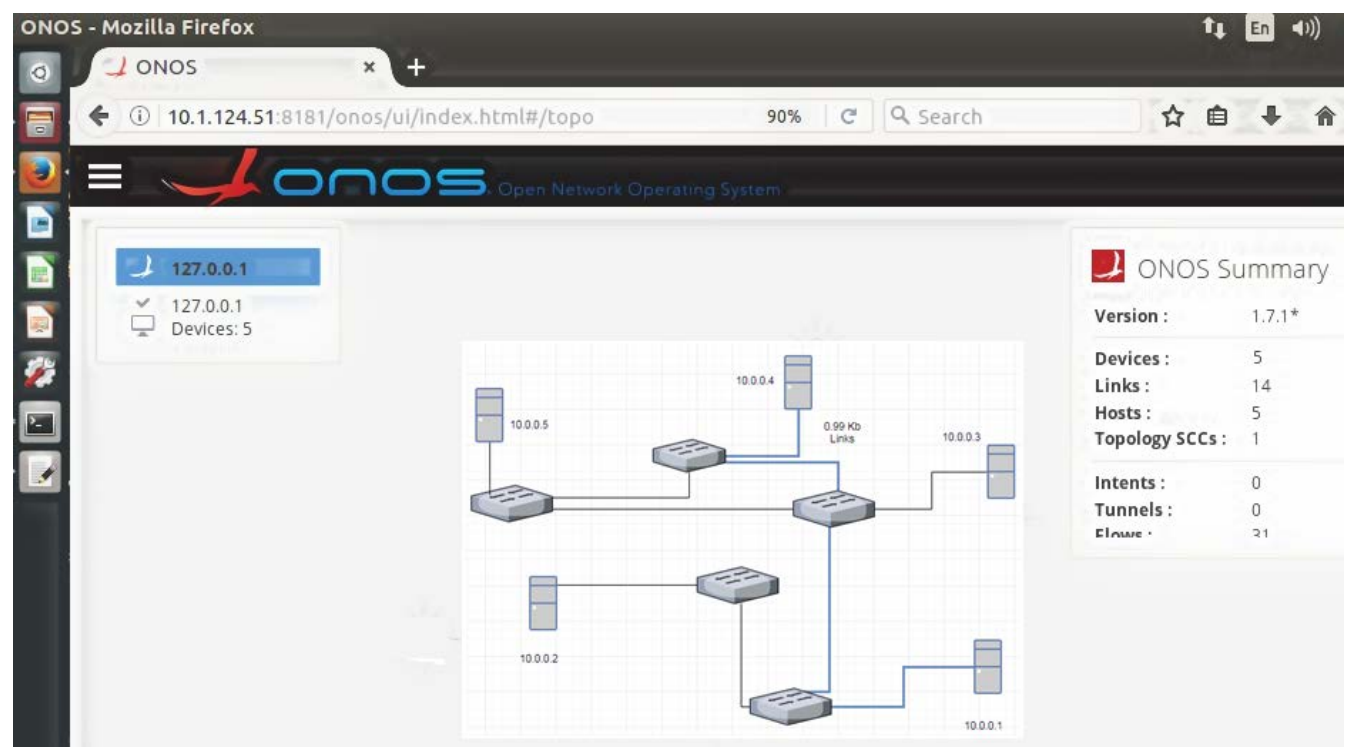

Fig. 8. ONOS environment with custom-made topology

\subsubsection{Executing the decision}

a) The PCE application will map the traffic demand with the appropriate child PCE instance (with the algorithm matched in step 6.1.2).

\subsection{Scenario: Creating Dynamic Elephant flows with Least Congestion}

We have considered the scenario of "dynamic elephant flow creation with least congestion". The ONOS CLI provides the ability to generate and transport traffic through the physical topology. The links were created between the switches and nodes with the predefined link attributes. In our work, we proposed to use multiple child PCE's (where each child PCE executing distinct RWA (ie path computation) algorithms). And the SDN controller will act as the parent PCE. The communication model and synchronization mechanism between the parent and child PCE's are well controlled by the SBI south bound interface (ie openflow protocol).

The child PCEs were programmed to compute the paths based on either the Constrained Shortest Path First (CSPF) algorithm or the Adaptive Least Congested algorithm (ALCA) [19][20]. The CSPF algorithm will compute the paths according to the specified set of constraints (ex: end-to-end delay, maximum number of hops, minimum required bandwidth, include/exclude nodes). The paths computed by CSPF could be exactly same as the one computed by either OSPF-Open shortest Path First or IS-IS-Intermediate System to Intermediate System or it could be varied according to the required set of constraints. The 
ALCA algorithm will compute the paths with minimal congestion. This algorithm will require the updated real time link status about the number of simultaneous on-going paths. This algorithm is mainly used to move the traffic towards the least congested route. Whenever the QoS requirements of the service mandates congestion this algorithm will be used. Hene both CSPF and ALCA algorithms are the existing algorithms used in the PCE based optical provisioning. As we considered the scenario of least congested path computation, we have considered the ALCA algorithm. For the simulation study we restricted our scope with only these two algorithms. The ONOS can monitor the switch updates through real time port and switch monitoring process. We have used PCE and PCECC-PCE Connection Controller applications to realize the GMPLS capability for the underlying network. We have dynamically activated bulk LSP demands each with different QoS requirements. Through the flow related commands each flow is monitored. We have written a java based NOX controller to coordinate between the PCC's and parent PCEs. Each time the PCC generates the requests with the different requirements like least congestion etc. The root PCE controller (orchestrator for the child PCEs) will dynamically select the best algorithm for the computation of paths. The CSPF and ALCA algorithms were implemented as child PCEs under the control of root/parent PCE Fig. 9.

PCE BASEDSDN CONTROL PLANE

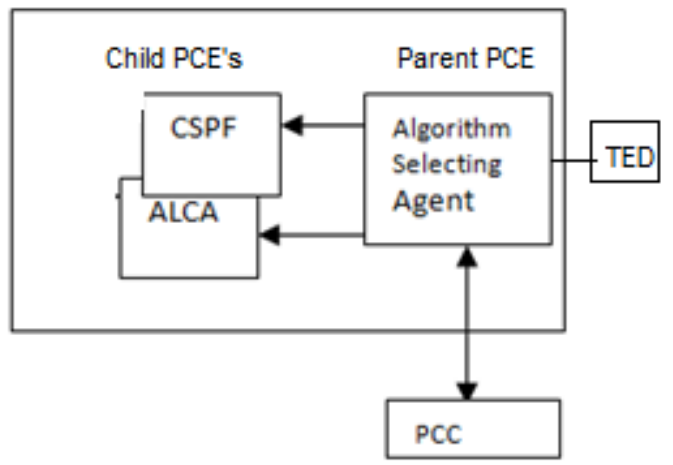

Fig. 9. Dynamic selection of PCE's with SDN

And the child PCEs (PCE replicas) will rely on the updated real-time LSP \& TED DB's of the parent PCE. With the packages like bgpflow and bgpcep the functionality of BGP and PCE were realized. One these applications were loaded, the custom written java application was executed to establish the dynamic tunnels with certain requirements.The distinctive PCE tunnels have been created intentionally using numerous QoS requirements including bandwidth, congestion, fee, and delay. We have made a custom topology with five nodes and five switches with the python script. Whenever the packet arrives at the router the flow tables entries will be matched and the appropriate action will be performed. If the packet does not match with any of the flow table entries then those requests will be sent to the controller for the appropriate action.

The link level details were observed for each LSP. The flow information had been observed to analyze the throughput and delay of the LSP's. The throughput (in Mb/s) and delay (in ms) of the established LSP's were monitored over a period of time. With tunnel based API's the state of the PCE and its tunnels were observed through diverse tunnel based API's. With centralized monitoring the controller can aggregate the level of congestion of each established 
LSP's between the two PCC's through the analysis of the port statistics (device wise). The flow table fields [9] that can be monitored by the ONOS controller is given below: Flow ID, Table ID, APP ID, Permanent, State, Group ID, Packets, Bytes, Priority, Timeout. These flow related informations can be generated as a JSON file and sent to the controller.

\subsubsection{LSP Tunnel Creation, Modification and Deletion}

The established tunnels were monitored through the ping command.Fig. 10.

The state of the links and tunnels were observed with the tunnel related commands. The following table shows the sample tunnel statistics Table 2. Through the tunnel API's the tunnels can be dynamically created, updated and deleted. During the tunnel updation additional flows can be added in addition to the existing flows. In all the tunnel related functions the source LSR, destination LSR and tunnel ID were quoted.

The following section explains the overall work flow of proposed work involved in the suggested configuration:

1. The LSR head end is termed as Path Computation Client (PCC) transfers a $P C_{\text {req }}$ message by the source IP, required bandwidth, destination IP, setup/holding priority, request priority and Traffic Engineering (TE)-Metrics to the server of Path Computation Element (PCE).

2. All the processes concerning to the parent path computation element (Parent-PCE) will be occurred inside of SDN controller and the satisfactory path will be computed as an effective manner.

3. The Algorithm Selection process in SDN controller will compute the another possible effective path apart from the satisfactory path, with the TE-Metric along with the exact algorithm of path computation. New child PCE occurrence will be created to calculate the enhanced LSPs. Depending upon the type of request the LSP's will be tuned thus to cater the required quality of service (QoS).

4. An optimal LSP will be updated in the flow table, after the computation of effective alternative path (LSP).

5. If the path is not effective then will not be reconfigured and the service will be continued in the satisfactory path itself. Then the computed effective path will be kept as backup to manage any unexpected anomalies with the network events/cable cut or device failures.

6. For a particular type of traffic trunk, the flow tables are kept updated and the LSP's will be forwarded under the control of the appropriate child PCE instance maintained with the SDN controller.

Hence in this manner, the different LSP's with different service classes of traffic will get mapped with the appropriate child PCE instances running different path computation algorithms. Hence the multiple hierarchal PCE's will be used to find the inter-domain paths where each child PCE is responsible for its own domain. In knowledge base, the optimal solutions will be stored and it will be used as future reference and on-demand path provisioning with minimum reconfiguration process. In general, the PCE based inter-domain routing architecture brings the capability to compute inter-domain routing with shortest constrained TE paths. In our proposed approach the PCE based inter-domain routing architecture brings the capability to execute multiple algorithms according to the features of the traffic demand and network status all while considering the negotiated QoS. 
In this way, our proposed work states that there will be multiple algorithms executed in parallel with the help of multiple child PCE instances as as to explore different search regions of the solution space. It was also wildely accepted that each algorithm or heuristic has its own potential to explore the search space.

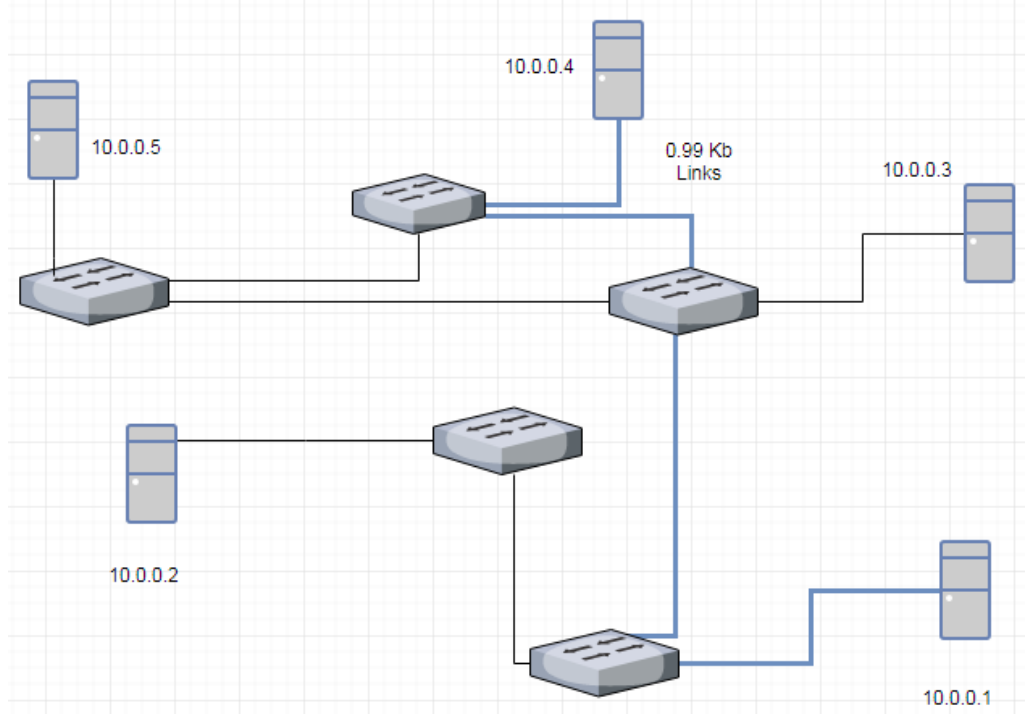

Fig. 10. Tunnel establishment in the custom 5 Node- 5 Switch optical topology

Table 2. Sample Tunnel details observed with tunnel monitoring

\begin{tabular}{|c|c|c|c|c|c|}
\hline LSP & $\begin{array}{c}\text { SOUR } \\
\text { CE } \\
\text { LSR ID }\end{array}$ & $\begin{array}{c}\text { DESTIN } \\
\text { ATION } \\
\text { LSR ID }\end{array}$ & $\begin{array}{c}\text { SOURCE- } \\
\text { INTERFA } \\
\text { CE }\end{array}$ & $\begin{array}{c}\text { DESTINA } \\
\text { TION- } \\
\text { INTERFA } \\
\text { CE }\end{array}$ & $\begin{array}{c}\text { TUNNEL } \\
\text { ID }\end{array}$ \\
\hline $\begin{array}{c}\text { CREA } \\
\text { TE }\end{array}$ & 4.4 .4 .4 & 5.5 .5 .5 & 11.1 .1 .4 & 11.1 .1 .5 & 1234 \\
\hline $\begin{array}{c}\text { UPDA } \\
\text { TE }\end{array}$ & 4.4 .4 .4 & 5.5 .5 .5 & 12.1 .1 .4 & 12.1 .1 .5 & 1234 \\
\hline
\end{tabular}

At any instance the status of the tunnels can be fetched from the Tunneldb. Through this database the state of the tunnel can be referred.

\section{Analysis of Results}

We have analyzed the throughput of the established LSP's to measure the efficiency of the proposed approach. We observed the total number of packets transmitted in a period of time from the tunnel $\mathrm{db}$. The port statistics was exported in the form of JSON file and the same is imported to the tableau tool plot the visual representation of the collected statistics. We have generated the packets of various sizes to test the proposed approach. The dynamic algorithm selection module was able to associate the created demands with the appropriate algorithms for the computation of paths. 


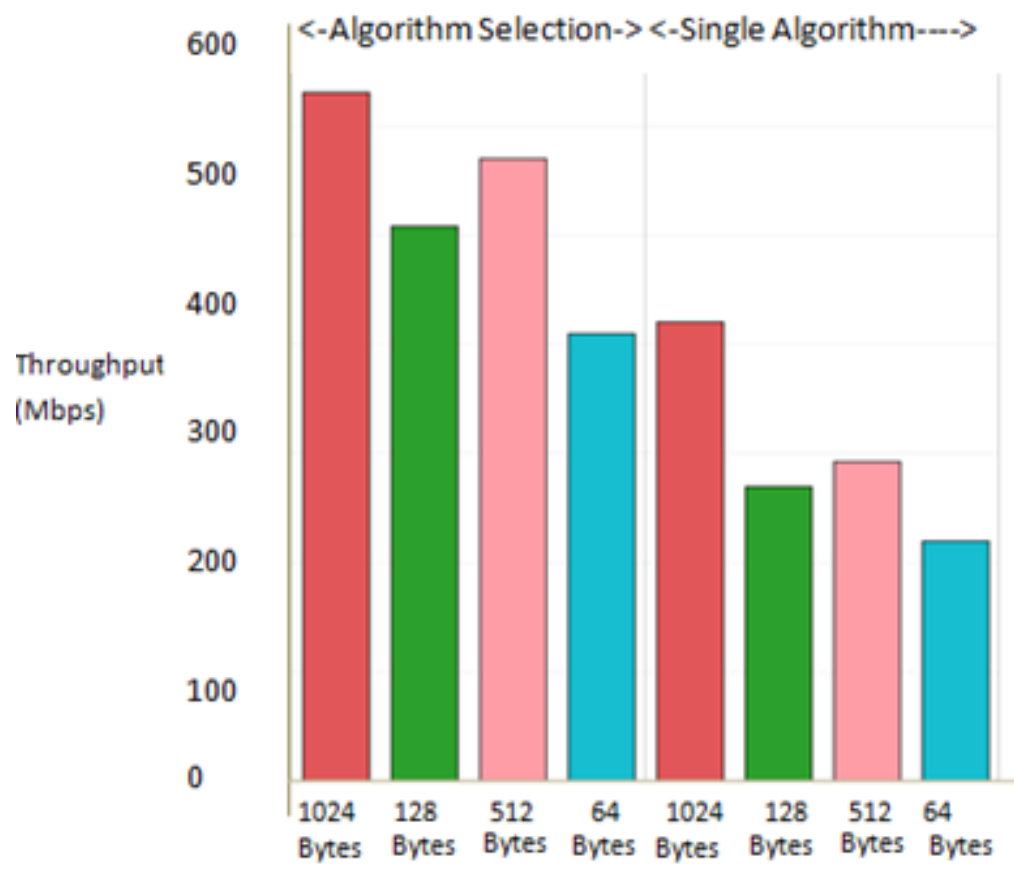

Fig. 11. Comparision of throughput between the single algorithm Vs algorithm selection based approach.

Table 3. Algorithm Slection Depending On SDN And PCE

\begin{tabular}{|c|c|c|c|c|}
\hline $\begin{array}{c}\text { Arrival } \\
\text { time }\end{array}$ & $\begin{array}{c}\text { \# } \\
\text { Source }\end{array}$ & \#Destination & $\begin{array}{c}\text { Capacity } \\
\text { (in } \\
\end{array}$ & $\begin{array}{c}\text { Duration } \\
\text { (in ms) }\end{array}$ \\
\hline 1.4 & 1 & 4 & 425.2 & 185 \\
\hline 2.2 & 3 & 1 & 278.6 & 120 \\
\hline 4.1 & 4 & 1 & 425.2 & 140 \\
\hline
\end{tabular}

The proposed algorithm selection model performs better in transporting packets of different sizes ranging from 64 bytes to 1024 bytes. The transportation of packets between the nodes with different sizes that ranges from 64 bytes to 1024 bytes has been performed. Therefore, the study of measures which indicates the efficacy of the network model was performed at the desired time interval. The statistics of the port was obtained from the JSON file. The presented algorithm selection model performs well when comparing the transferring of packets that ranges from 64 to 1024 bytes which indicates the efficacy of the network model. The five node optical topology was created in the ONOS environment for simulating the dynamic elephant flows transportation Table 3 with the least congestion as much as possible. The elephant flow creation were made with the gaussian distribution model. The traffic 
between nodes were created using the poission distribution model Table 4. The throughput analysis is the important measure that indicates the efficiency of the network model. The throughput was measured over a time period. The result have shown that the dynamic PCE selection approach performs much better than the single algorithm based provisioning in-terms of the throughput Fig. 11.

Table 4. Node Network Traffic Generation for the simulation

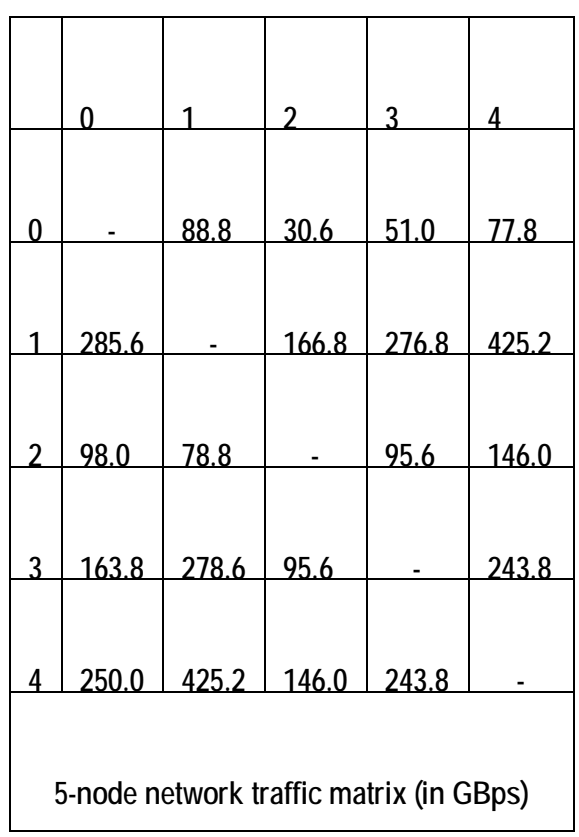

The five node optical topology was created in ONOS based environment to simulate the scenario of dynamic elephant flow creation with least possible congestion. The controller was able to find the appropriate algorithm to compute the path based on the algorithm selection model. In the case of least congested path establishment the ALCA algorithm was selected as the algorithm for the PCE.

\section{Conclusion}

Hence a novel dynamic algorithm selection methodology was proposed to solve the dynamic path establishment problem in optical networks. The SDN's centralized control and mangement ability has motivated the innovation in the way of path provisioning. This paper has proposed a match field based algorithm selection as a simple yet effective technique. The dynamic path provisioning in the next generation optical network would involve different QoS mandates according to the type of application. The dynamic voluminous demands could be provisioned effectively with the help of dedicated PCE underneath the control of the SDN controller. To manage the multiple algorithms simulataneously in the same operator domain the parent-child based PCE management approach was proposed. In this way every child PCE can execute different algorithms. The root/parent PCE will coordinate between the child PCE's in porting the traffic with the concern child PCE's. An SDN's packet matching feature inspired algorithm match field technique was proposed as an algorithm selection method. And the SDN based dynamic PCE selection approach was claimed better than the single algorithm 
based provisioning in-terms of the throughput. As reducing the network latency and increasing network throughput are the most fundamental tasks in network designs the proposed model was evaluated with various type and sizes of network demands with dedicated PCE. To prove the efficiency of this method an ONOS based simulation has been performed. From the simulation study it was evident that the dynamic algorithm selection module was capable of selecting the appropriate algorithm for the path computation with the consideration of network state and the type of the traffic flow. The use case of bulk LSP creation, deletion and modification with varying QoS specification was demonstrated. The tunnel, flow and packet level statistics were observed to find the throughput and latency of the proposed algorithm selection approach. And the proposed approach was claimed as an ameanable one for the next generation optical systems.

\section{Limitations}

This paper is in lack of accounting the communication latency of the parent-child system. The communication latency of the PCE based system involves two aspects 1) PCE's path computation latency and 2) the control plane processing latency. Firstly we assumed that the SDN has abstracted the hidden complexities behind virtualization and dynamic path provisioning. The delay involved in orienting the appropriate algorithm inside the SDN controller was assumed minimum, as the proposed model follows the match fields based lookup approach. But there will be a delay involved with the path computation latency, which is the out of scope of this paper. Secondly the control plane processing latency is the time taken to establish the secured communication channel and transporting the control instructions. There will be some considerable initial latency in establishing a secure channel between the PCC and PCE. We assumed that the delay will be incurred only at the very first time when the PCC nodes are booted and get discovered by the PCE and there onwards there will be an abstracted communication channel maintained to provide the necessary logical abstractions. Hence these are the two aspects which were considered as out of scope of this paper.

\section{Future Work}

As a continuation of this work, we have planned to develop a dynamic feature based grouping of algorithms with the real emulated demonstration in the SDN test bed. The accurate pattern recognition and generalization ability of neural network based deep learning will be exploited to create an intelligent and dynamic algorithm selection model.

\section{References}

[1] Borkowski R, Durán RJ, Kachris C, Siracusa D, Caballero A, Fernández N, Klonidis D, Francescon A, Jiménez T, Aguado JC, de Miguel I., "Cognitive optical network testbed: EU Project CHRON," Journal of Optical Communications and Networking, 7(2), p. 344-355, 2015. Article (CrossRef Link)

[2] Alvizu R, Maier G., "Can open flow make transport networks smarter and dynamic? An overview on transport SDN," in Proc. of InSmart Communications in Network Technologies (SaCoNeT), 2014 International Conference, pp. 1-6, IEEE, 2014. Article (CrossRef Link)

[3] Omnes N, Bouillon M, Fromentoux G, Le Grand O., “A programmable and virtualized network \& IT infrastructure for the internet of things: How can NFV \& SDN help for facing the upcoming challenges," in Proc. of InIntelligence in Next Generation Networks (ICIN), 2015 18th International 
Conference, pp. 64-69, IEEE, 2015. Article (CrossRef Link)

[4] Mu-oz R, Vilalta R, Casellas R, Martinez R, Szyrkowiec T, Autenrieth A, et al., "Integrated SDN/NFV management and orchestration architecture for dynamic deployment of virtual SDN control instances for virtual tenant networks," Journal of Optical Communications and Networking, vol. 7,no. 11, pp. B62-B70, 2015. Article (CrossRef Link)

[5] Thyagaturu AS, Mercian A, McGarry MP, Reisslein M, Kellerer W., "Software defined optical networks (SDONs): A comprehensive survey,” IEEE Communications Surveys \& Tutorials, 18(4), pp.v2738-2786, 2016. Article (CrossRef Link)

[6] Oliveira J, Oliveira J, Magalhães E, Januário J, Siqueira M, Scaraficci R, et al, "Toward terabit autonomic optical networks based on a software defined adaptive/cognitive approach,” Journal of Optical Communications and Networking, vol. 7,no. 3, pp. A421-A431 2015.

Article (CrossRef Link)

[7] Varvarigos EA \& Christodoulopoulos K, "Algorithmic aspects in planning fixed and flexible optical networks with emphasis on linear optimization and heuristic techniques," Journal of Lightwave Technology, vol. 32,no. 4, pp. 681-693, 2014. Article (CrossRef Link)

[8] Bhatt S \& Jhaveri S, "A review of dense wavelength division multiplexing and next generation optical internet,” International Journal of Engineering Science and Innovative Technology (IJESIT) Volume, vol. 2, 2013. Article (CrossRef Link)

[9] Yoshida Y, Maruta A, Kitayama K-i, Nishihara M, Tanaka T, Takahara T, et al, "SDN-based network orchestration of variable-capacity optical packet switching network over programmable flexi-grid elastic optical path network," Journal of Lightwave Technology, vol. 33,no. 3, pp. 609-617, 2015. Article (CrossRef Link)

[10] Filer M, Gaudette J, Ghobadi M, Mahajan R, Issenhuth T, Klinkers B, et al, "Elastic optical networking in the Microsoft cloud," Journal of Optical Communications and Networking, vol. 8,no. 7, pp. A45-A54, 2016. Article (CrossRef Link)

[11] Aguado A, López V, Marhuenda J, de Dios ÓG \& Fernández-Palacios JP, “ABNO: A feasible SDN approach for multivendor IP and optical networks," IEEE/OSA Journal of Optical Communications and Networking, vol. 7,no. 2, pp. A356-A362, 2015. Article (CrossRef Link)

[12] Buchler N, Marusich LR \& Sokoloff S, "The Warfighter Associate: Decision-support software agent for the management of intelligence, surveillance, and reconnaissance (ISR) assets," Ground/Air Multisensor Interoperability, Integration, and Networking for Persistent ISR V, p. 907902, 2014. Article (CrossRef Link)

[13] Chen C, Chen X, Zhang M, Ma S, Shao Y, Li S, et al, "Demonstrations of efficient online spectrum defragmentation in software-defined elastic optical networks," Journal of Lightwave Technology, vol. 32,no. 24, pp. 4099-4109, 2014. Article (CrossRef Link)

[14] Lara A, Kolasani A, Ramamurthy B, "Network innovation using OpenFlow: A survey," IEEE communications surveys \& tutorials, 16(1), pp. 493-512, 2014. Article (CrossRef Link)

[15] Chen X \& Zhang Y, "Intelligence on optical transport SDN," International Journal of Computer and Communication Engineering, vol. 4,no. 1, p. 5, 2015. Article (CrossRef Link)

[16] Cvijetic N, Tanaka A, Kanonakis K \& Wang T, "SDN-controlled topology-reconfigurable optical mobile fronthaul architecture for bidirectional CoMP and low latency inter-cell D2D in the 5G mobile era,” Optics express, vol. 22,no. 17, pp. 20809-20815, 2014. Article (CrossRef Link)

[17] Berde P, Gerola M, Hart J, Higuchi Y, Kobayashi M, Koide T, Lantz B, O'Connor B, Radoslavov P, Snow W, Parulkar G., “ONOS: towards an open, distributed SDN OS," in Proc. of Proceedings of the third workshop on Hot topics in software defined networking, ACM, pp. 1-6, 2014. Article (CrossRef Link)

[18] Gkamas V, Christodoulopoulos K \& Varvarigos E, “A joint multi-layer planning algorithm for IP over flexible optical networks,” Journal of Lightwave Technology, vol. 33,no. 14, pp. 2965-2977, 2015. Article (CrossRef Link)

[19] Zhou B, Mouftah HT, "Adaptive least loaded routing for multi-fiber WDM networks using approximate congestion information," in Proc. of InCommunications, 2002. ICC 2002. IEEE International Conference on 2002, Vol. 5, pp. 2745-2749, IEEE, 2002. Article (CrossRef Link) 
[20] Jiang D, Xu Z, Li W \& Chen Z, "Network coding-based energy-efficient multicast routing algorithm for multi-hop wireless networks," Journal of Systems and Software, vol. 104, pp. 152-165, 2015. Article (CrossRef Link)

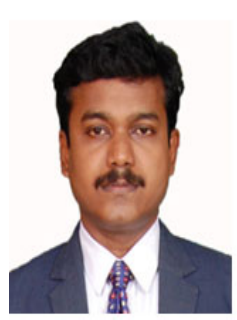

P.Selvaraj is working a Senior Assistant Professor at SRM Institue of Science and Technology, Chennai, Tamilnadu, India. He has been in teaching past 13 years. He constantly handled the subjects related to aritificial intelligence and machine learning. He did several online certifications like R programming, Machine learning, AI programming etc., He secured a proxor certification in java. He has contributed for the AI modules of university projects which involves MATLAB, SOAR, R programming. He published 14 indexed journals in the fields of cognitive optical networks, image processing, machine learning. He is serving for the university by organizing various technical lectures and promoting research among students. Currently he is working in the field of next generation intelligent optical networking using AI strategies. He is a part of research team in 'software defined optical test bed lab facility' which is established in SRM institue in association with Tejas Networks, Bangalore.

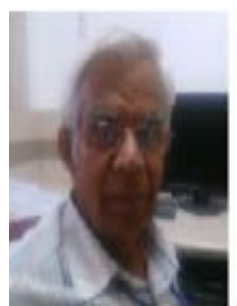

Dr.V.Nagarajan is currently the chairman of Andromedia communication(P)Ltd., Pune. He has been in the field of optical communication research for the past 44 years. He completed his $\mathrm{PhD}$ (Elec) in Indian Institute of Technology, Delhi , 1972-'73. He worked in top cadre positions in both government and private sectors. Some of his work experience includes Indian Institute of Technology, Delhi (lecturer), Ericsson Telephone AB-Sweden (System Manager), Optel Telecommunications Ltd, Bhopal (CEO), Indian Telephone Industries, Bangalore (Deputy Chief Engineer). He authored 2 books in optical networking. He has many honors which includes Fellow of National Academy of Engineering, Fellow IETE, India, Member IEEE, USA, Member, New York Academy of Science, representative of International standards committees like ITU, ANSI, Expert committee of NBA under AICTE. He has been constantly involving in Industry-Institute Interaction, Network Construction and Management, Strategic Business Development, Project-Consultancy. 